\title{
VERIFICATION OF THE DIGITAL IMAGE CORRELATION TECHNIQUE FOR BONE SURFACE STRAIN MEASUREMENTS
}

\author{
Rajesh Ghosh (1), Sanjay Gupta (1), Alexander Dickinson (2), Martin Browne (2) \\ 1. Indian Institute of Technology Kharagpur, India; \\ 2. University of Southampton, United Kingdom
}

\section{Introduction}

Digital image correlation (DIC) has been used on composite bone structures to evaluate surface strain patterns before and after implantation [Tayton et al. 2010, Dickinson et al. 2011]. However, there is a lack of experimental data on full field strain measurement on biological tissue. The aim of the study was to test the validity of DIC measurements on bone by comparing DIC measured strain with strain rosette data on an ovine femur specimen.

\section{Methods}

Two separate experiments were carried out on a fresh ovine femur specimen (Fig. 1). Soft tissues were removed from the bone surface, which was degreased with acetone and ethyl alcohol. The distal end of the femur was resected to allow potting and fixation to a base plate. The DIC measurements were carried out prior to the strain rosette measurements on the same specimen, for one-to-one comparison of data. The specimen was supported and loaded identically in a servohydraulic Instron testing machine (Instron 8874, Instron Corp., Norwood MA, USA, load capacity $\pm 25 \mathrm{kN})$. Random speckle patterns were applied on the bone surface with an air brush. The displacement of the speckle patterns due to load application were recorded using 2MP digital cameras (Limess GmbH, Germany), and VIC 3D correlation software (Correlated Solutions Inc., SC, USA) was used to calculate the strain field. After removal of the white paint and the speckle patterns from the bone surface with acetone and alcohol, four strain rosettes were

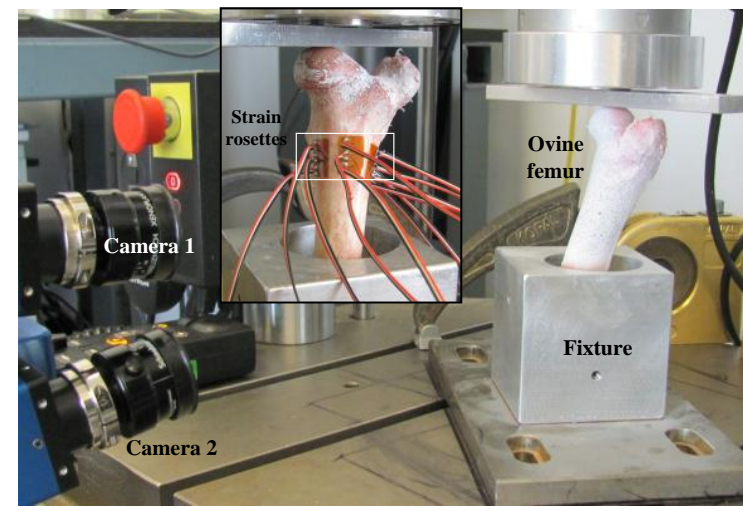

Figure 1: Experimental set-up for DIC and strain rosette (inset) measurement using ovine femur. fixed at four locations on the proximal femoral shaft - medially, anteriorly, anterior-laterally and laterally (Fig. 1). Compressive hip-joint reaction forces of $1000 \mathrm{~N}, 1200 \mathrm{~N}$ and $1400 \mathrm{~N}$ were applied to the femoral head. Three successive experiments were performed to quantify the measurement repeatability. Three repeated loaded datasets from successive experiments resulted in six sigma experimental error ( \pm three standard deviations) of $< \pm 43 \mu \varepsilon$ in the medial area, $< \pm 59 \mu \varepsilon$ in the anterior area, $< \pm 19 \mu \varepsilon$ in the anterior-lateral area and $< \pm 65 \mu \varepsilon$ in the posterior area of the proximal femoral shaft.

\section{Results and Discussion}

Regression analysis indicated a strong linear relationship between the DIC and strain rosette measurements, with a high correlation coefficient $(\mathrm{R}=0.99)$, a low standard error of the estimate (SE $=33 \mu \varepsilon$ ) and regression slope of 1.05. The DIC and strain rosette measurements indicated predominantly compressive strain at the medial

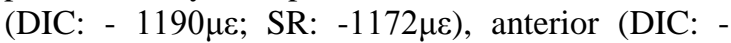
$436 \mu \varepsilon$; SR: $-417 \mu \varepsilon$ ) and lateral (DIC: $-180 \mu \varepsilon$; SR: - $100 \mu \varepsilon$ ) locations. However, predominantly tensile strain was measured at the anterior-lateral (DIC: $216 \mu \varepsilon$; SR: $202 \mu \varepsilon$ ) location of the proximal femoral shaft. Although close agreement was observed between the DIC and strain rosette measurements, some deviation was observed at highly curved boundaries. The main caveat, which is not captured by this validation, is that great care should be taken in removing soft tissue from the bone surface, to ensure that it is not affected mechanically.

\section{Conclusions}

This study demonstrated close agreement between the established strain gauging technique and Digital Image Correlation on ovine bone. This supports the validity of DIC measurements to obtain full-field strain data on biological specimens.

\section{References}

Tayton et al, JBJS, 92:1176-1181, 2010.

Dickinson et al, J Biomech Eng, 133:1-6, 2011.

\section{Acknowledgement}

The authors are thankful to UKIERI British Council for supporting this study. 